\title{
Target Tracking And Energy Optimization Based On Dynamic Clusters Mechanism In WSN
}

\author{
Qun Zhuang ${ }^{1, \text { a }}$, Zibo $\mathrm{He}^{2, \mathrm{~b}}$ \\ ${ }^{1}$ QingDao Ocean Shipping Mariners College, QingDao, China \\ ${ }^{2}$ Weichai Power Co., Ltd is,WeiFang China \\ aZhuangqun@coscoqmc.com.cn, bhezibo1987@163.com
}

\begin{abstract}
Keywords: Dynamic Cluster; Energy Optimization; Target tracking;
Abstract. In order to reduce the entire network sensor node energy consumption, this text gives an algorithm to track the target effectively and to reduce the energy using the uniform speed and variable speed motion target tracking in Wireless sensor networks as the background. In addition, in order to ensure the accuracy, dynamic members of the cluster nodes using the maximum likelihood to calculate and track the target. Detection radius of sensor node classification to reduce the energy consumption of the entire network. Simulation results show that the algorithm can achieve the accuracy of target tracking and optimize of the network energy consumption. This template explains and demonstrates how to prepare your camera-ready paper for Trans Tech Publications. The best is to read these instructions and follow the outline of this text.
\end{abstract}

\section{Introduction}

This paper presents an optimization algorithm based on dynamic cluster target tracking mechanism and energy optimization. The tracking algorithm is based on the RSSI strength of cluster head selection mechanism, all nodes are in a dormant state in the case of no target entry, in the sub-cluster, the node 's residual energy and the target signal intensity factor is the decisive factor in the cluster head selection. Cluster head in such target tracking is the node that network energy stronger and easier to track the target, so the network robustness enhanced, with the target moves, the cluster structure will be dynamically adjusted to ensure that the network will target tracking. How to ensure tracking carried out under the premise that effective to reduce network energy consumption is a key issue in wireless sensor network-based target tracking algorithm. This article assumes that the target loss process, the network regain the energy consumption of the test target optimization proposed an energy efficiency of the algorithm, In this method, using dynamic control the radius, the detection radius of the sensor nodes can effectively reduce energy consumption.

\section{Classic target tracking algorithm}

WSN target tracking include the single-target tracking and multi-target tracking, this paper studies the single-target tracking [1]. Here are several classic single-target tracking algorithm. We choose transmission tree tracking algorithm as an example.

Static centralized routing tracking information is sent to the fixed cluster head to process. This approach often lead to results that the fixed cluster head energy consumption is too large, the network lifetime can not be guaranteed [2]. Transmission tree-based tracking algorithm is a distributed algorithm, the cluster head is dynamic, so you can effectively reduce energy consumption, ensure the whole network life $[3,4]$.

Transmission tree is that the nodes which near a moving target that form a dynamic tree structure. As the target moves, dynamic tree changes, add and remove some of the sensor nodes [5]. Dynamic tree nodes collaborate together to accomplish the target tracking. Figure 1 is the process of transmission tree tracking algorithm. When the target enters the detection area, the node who detect the target select a root node and construct transmission tree. Other nodes of the transmission tree will transmit the track data to the root node, then the root node store the message, and it will give the result to the sink node. 
During the moving, the tree structure dynamically remove the nodes far away from the target, and add the node that near the target. When the target and the root node exceed a certain threshold, the node will reconstruct the transmission tree.

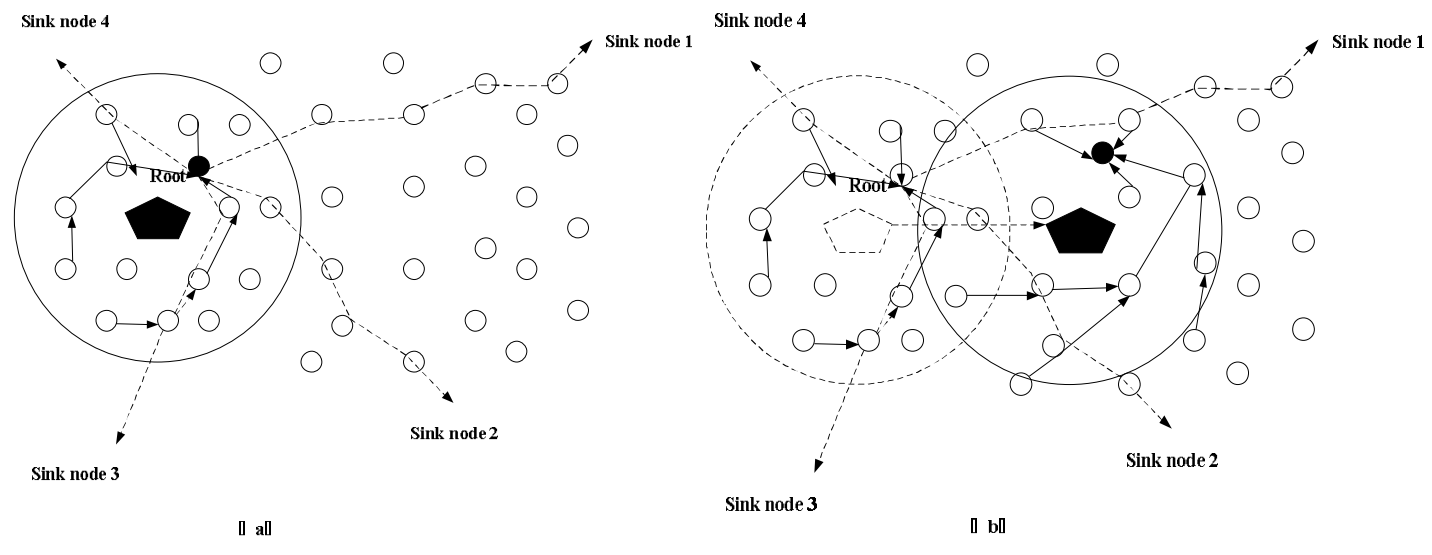

Figure 1. Target tracking based on dynamic transmission tree

In order to save energy of sensor nodes, sensor networks will use the clustering structure of the grid. Nodes in the cluster will be a cluster head node periodically. When the network does not detect the target, only the cluster head is in working condition, and ordinary node is sleeping. When the moving target enters into the net, the cluster head node will wake up other nodes in the cell responsibly [6].

\section{Improved target tracking and energy consumption optimization algorithm}

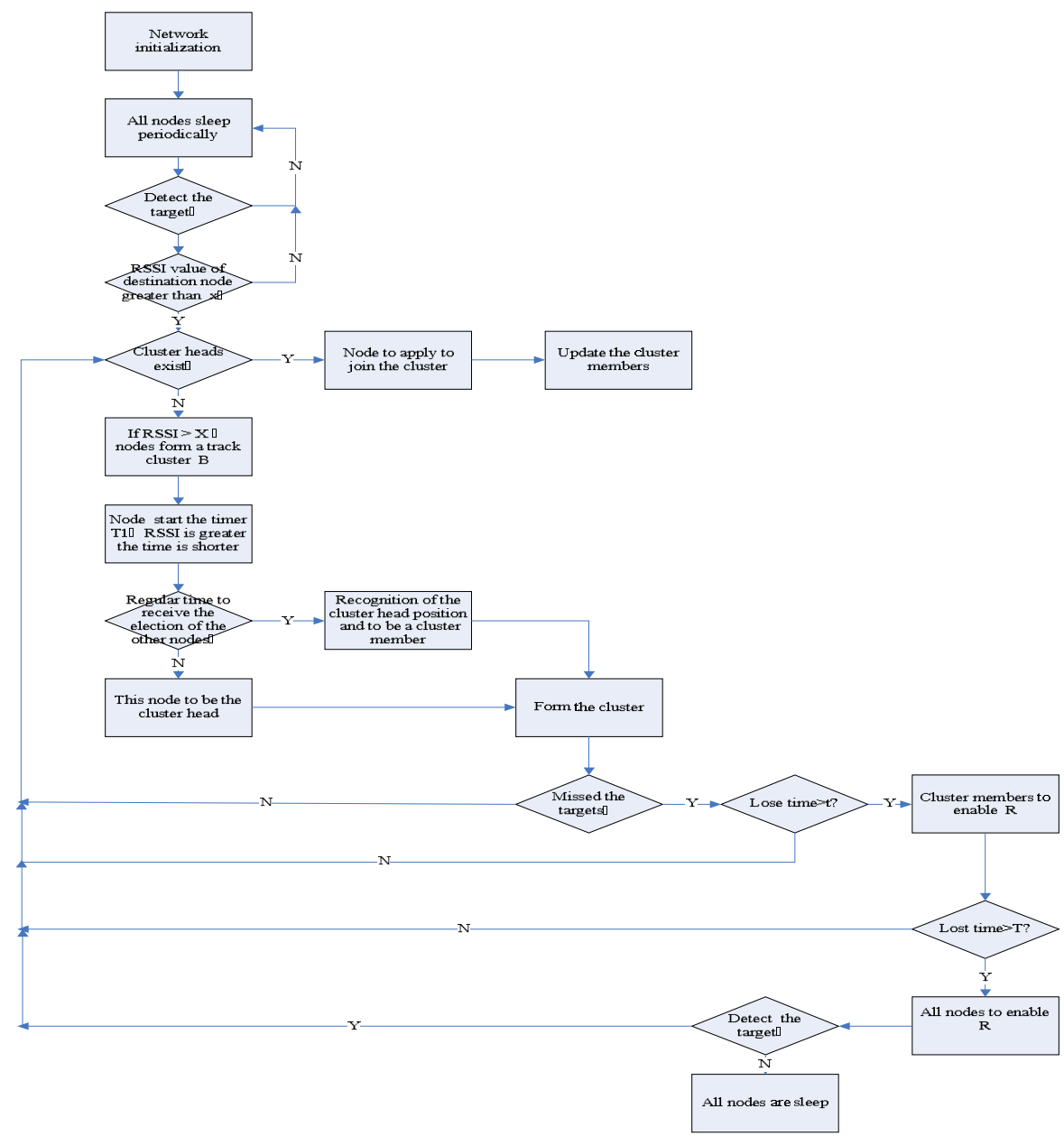

Figure 2. Flow chart

In this paper, the dynamic control of the detection radius of the sensor nodes, each sensor node are set a low power radius (r) and a high power $\operatorname{radius}(\mathrm{R})$, in normal circumstances, the node uses 
the power of perception radius $\mathrm{r}$. Any prediction algorithm there is a certain error, the cluster head node can set the time threshold $\mathrm{t}$ and $\mathrm{T}$, when time $\mathrm{t}$ has not yet received the target location information, it means that the goal has lost, and then the cluster head can start the recovery machine, nodes that in the cluster start high-power detection radius. Dynamic cluster node start the recovery mechanism, the current cluster member node using high-power sensing radius $\mathrm{R}, \mathrm{T}$ time , all nodes using high-power sensing radius $\mathrm{R}$, so that the detection area on the expansion of $\mathrm{R}$ is radius of the circular area that if it contains more nodes, it will greatly increase the possibility to recover the target. While this algorithm ensures the protection of target tracking, it also can effectively control the energy consumption of the entire network, thus ensure the survival of the entire network cycle. Simulation to simplify the system model as follows:

1) Node space deployment density and random, the coordinates (x, y) is known;

2) Each node has a unique ID, a data fusion function;

3) The node is isomorphic to a peer, the same range of wireless propagation;

4) The node has the ability to group clusters;

5) The node with the radius of the detection of high and low power.

Initial clusters create a flow chart shown in Figure 2.

Target and forecast are as follows:

After the initial cluster formation, adding to the definition of nodes in the cluster members for the collection $D=\left\{D_{1} \cdots D_{m}\right\}$, the moment of the target coordinates ( $\left.\mathrm{x}, \mathrm{y}\right)$, member nodes in the cluster periodically detect the target RSSI strength converted to the corresponding distance d, chronological record in its own memory, and then sent to the cluster head, cluster head node i according to the cluster members of the location information and distance $\mathrm{d}$, the maximum likelihood estimation method to locate the target position.

$$
d_{j}=d_{0} \cdot 10^{\frac{P\left(d_{0}\right)-P\left(d_{j}\right)}{10 n}}
$$

And then use the maximum likelihood estimate of the localization in the current location of the target. The target prediction is to predict the position and velocity of the target in the next time. Set goal movement curves obey the following polynomial function distribution:

$$
p_{n}(x)=\sum_{j=0}^{n} a_{j} x^{j}
$$

Take the minimum of the following type:

$$
R=\sum_{i=0}^{m}\left[p_{n}\left(x_{i}\right)-y_{i}\right]^{2}=\sum_{i=0}^{m}\left[\sum_{k=0}^{n} a_{k} x_{i}^{k}-y_{i}^{2}\right]^{2}=\min
$$

it may close to the actual function of distribution, as

$$
\begin{aligned}
& \frac{\partial R}{\partial a_{i}}=2 \sum_{i=0}^{m}\left(\sum_{k=0}^{n} a_{k} x_{i}^{k}-y_{i}\right) x_{i}^{j}=0 \\
& \left(\begin{array}{cccc}
m+1 & \sum_{i=0}^{m} x_{i} & \mathrm{~L} & \sum_{i=0}^{m} x_{i}^{n} \\
\sum_{i=0}^{m} x_{i} & \sum_{i=0}^{m} x_{i}^{2} & \mathrm{~L} & \sum_{i=0}^{m} x_{i}^{n+1} \\
\mathrm{M} & \mathrm{M} & \mathrm{M} & \mathrm{M} \\
\sum_{i=0}^{m} x_{i}^{n} & \sum_{i=0}^{m} x_{i}^{n+1} & \mathrm{~L} & \sum_{i=0}^{m} x_{i}^{2 n}
\end{array}\right) \cdot\left(\begin{array}{c}
a_{0} \\
a_{1} \\
\mathrm{M} \\
a_{n}
\end{array}\right)=\left(\begin{array}{c}
\sum_{i=0}^{m} y_{i} \\
\sum_{i=0}^{m} x_{i} y_{i} \\
\mathrm{M} \\
\sum_{i=0}^{m} x_{i}^{n} y_{i}
\end{array}\right)
\end{aligned}
$$

$a_{k}$ mean the function of the target distribution, thus predict the location of the next moment. 


\section{Simulation and Results}

In order to evaluate the proposed group clustering algorithm, I use Matlab to simulate the algorithm. First establish the two-dimensional target motion model. Assuming that the target movement in the two-dimensional space, rectangular area of the sensing area $(0 \mathrm{~m}, 0 \mathrm{~m})$ to $(200 \mathrm{~m}$, $200 \mathrm{~m}$ ).Algorithm parameter settings shown in TABLE 1.

Table 1

PARAMETER LIST

\begin{tabular}{|l|l|l|}
\hline parameter & describe & value \\
\hline$R_{N}$ & Communication radius & $40 \mathrm{~m}(\mathrm{r}) / 60 \mathrm{~m}(\mathrm{R})$ \\
\hline$R_{s}$ & target effective transmission radius & $20 \mathrm{~m}$ \\
\hline$e_{k}$ & node residual energy & $100 \%$ \\
\hline$\alpha, \beta$ & weighting factor & 1,1 \\
\hline$\varepsilon$ & transfer factor of cluster head & 0.4 \\
\hline $\mathrm{N}$ & the number of nodes & 500 \\
\hline$\Delta t$ & sampling period & $1 \mathrm{~s}$ \\
\hline
\end{tabular}

Due to the randomness of the distribution of nodes in the simulation process, it may lead to the difference of the number of cluster members within a region, and it will impact the complexity of simulation algorithm (cluster members is greater than ten) and accuracy(cluster members is less than three), Therefore, the provisions of the number of cluster members is between 3 and 6 .

\section{A. Target tracking error}

Uniform motion and variable speed moving target tracking simulation:

1) Uniform motion trajectory simulation:

$$
\left\{\begin{array}{l}
x=t \\
y=t
\end{array} .\right.
$$

Figure 3 sampling time $\mathrm{t}=100$ s cluster organization, which the black straight line for the real target trajectory, the black cross points are the ordinary nodes, red cross points are the campaign cluster member node, the blue circle points are the cluster member nodes, the black plus sign points are the target tracking trajectory. It can be seen in the process that the formation of clusters nodes are added to the network by the weighted value.

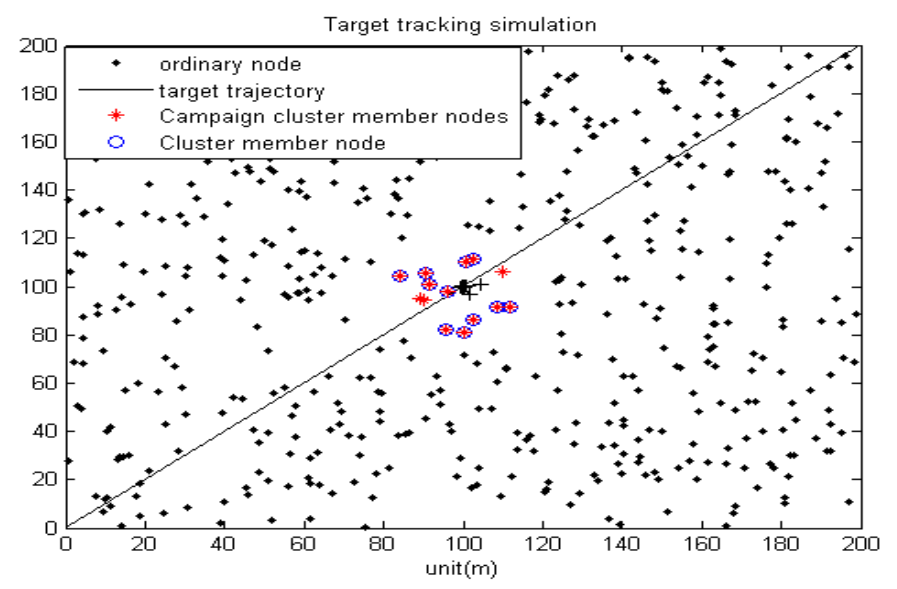

Figure 3. $\mathrm{t}=100 \mathrm{~s}$ when the tracking simulation

After many experiments, calculate the average error of this algorithm, the constant velocity target tracking error is $0.3644 \mathrm{~m}$, as shown in Figure 4. 

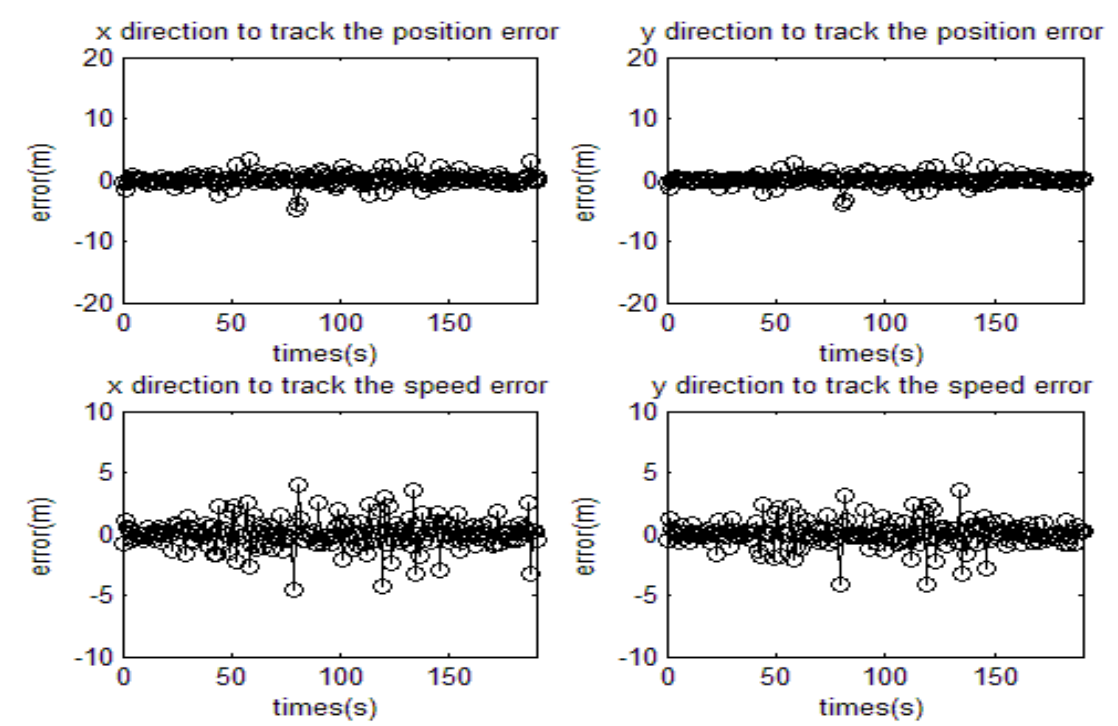

Figure 4. Uniform motion tracking error map

2) Variable motion trajectory simulation:

$$
\left\{\begin{array}{c}
x=t \\
y=1 / 40000 *(t / 10)^{\wedge} 3
\end{array}\right.
$$

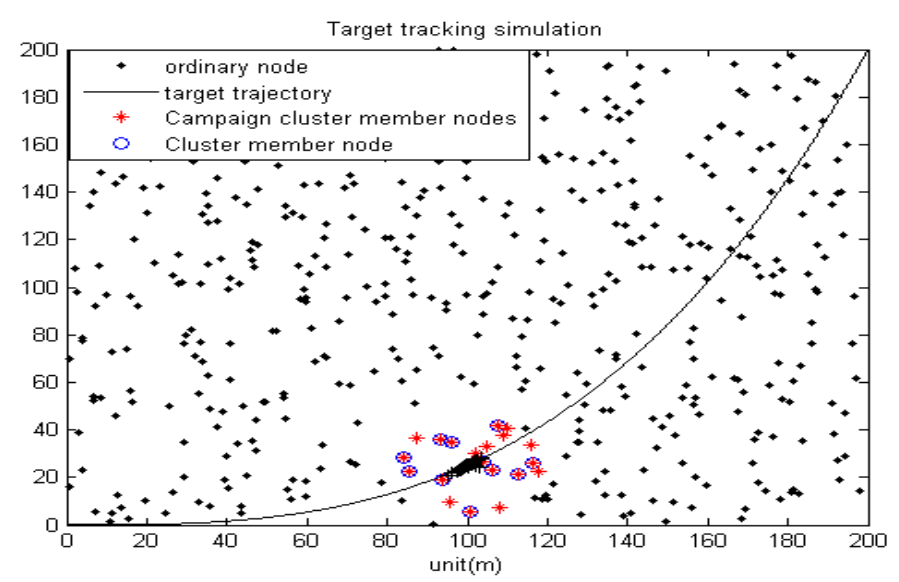

Figure 5. $t=100 \mathrm{~s}$ when the tracking simulation
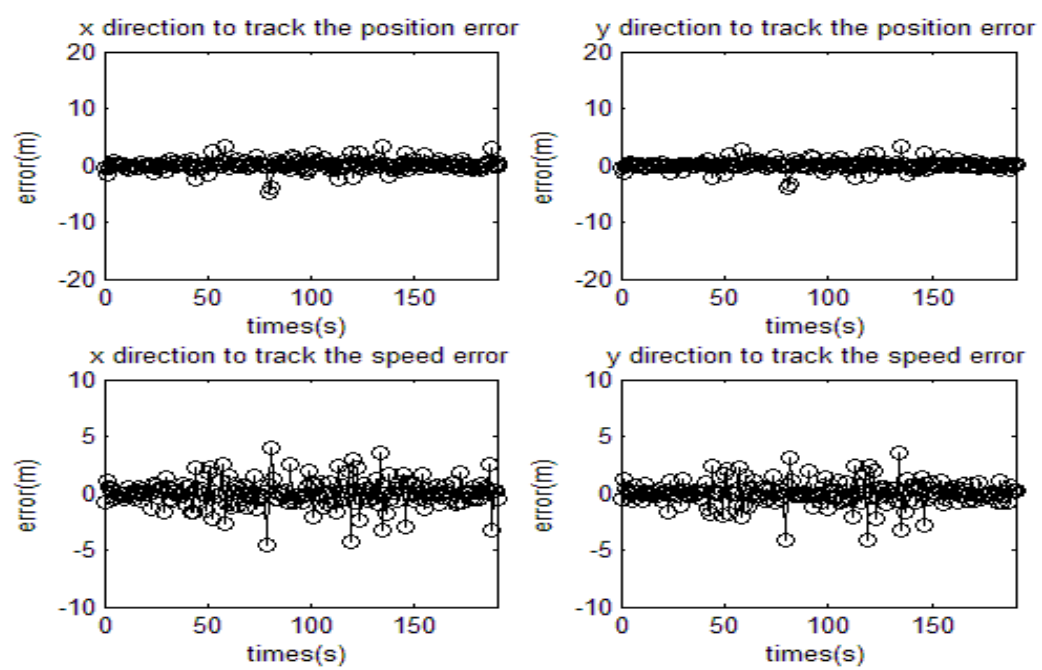

Figure 6. Variable motion tracking error

Figure 5 sampling time $\mathrm{t}=100 \mathrm{~s}$, the black plus sign points are the target tracking trajectory; It can be seen in the process that the formation of clusters nodes are added to the network by the 
weighted value. After many experiments, calculate the variable speed moving target tracking average error is $0.47885 \mathrm{~m}$ in this algorithm, for examples shown in Figure 6.

Through the simulation it can be seen that in the process of constant speed and variable speed target tracking, the algorithm maintains good performance.

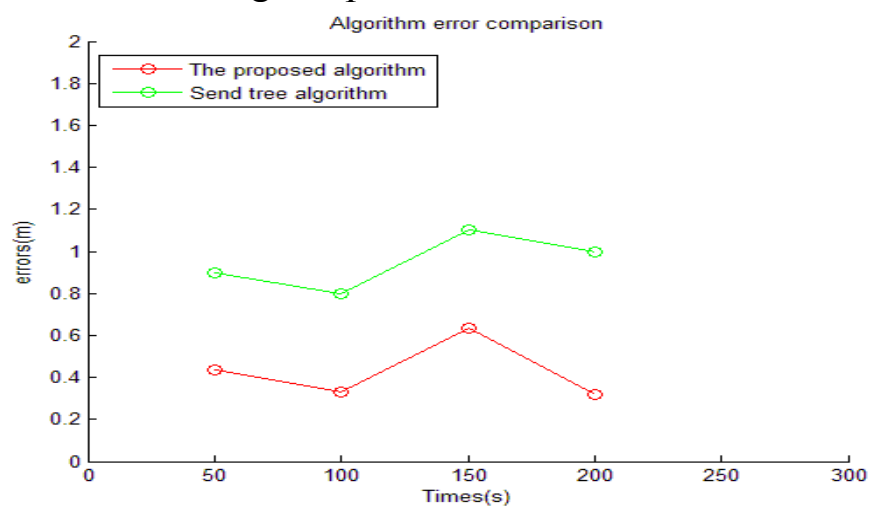

Figure 7. Comparison of tracking error

Figure 7 shows the error between the two algorithms, after a large number of the simulation we can come to these conclusions. Error curve of the proposed algorithm in which the red, green shows the traditional transmission tree tracking algorithm error curve. It can be seen that this algorithm can achieve the tracking of moving targets on the tracking error to meet the requirements.

B. Distribution number on the tracking error

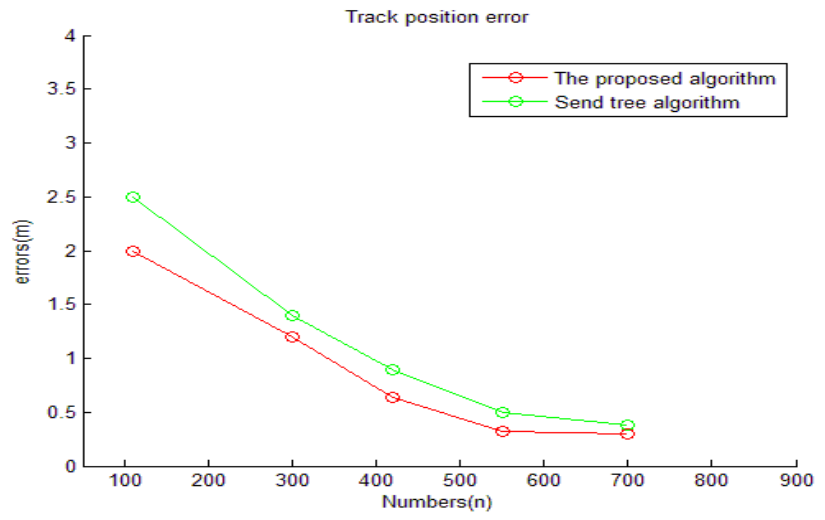

Figure 8. Distribution number on the tracking error

After a number of simulation experiments, as it shown in Figure 8, we get the diagram of the number of distribution and tracking error. Tracking error of the red for the proposed algorithm, Green said the tracking error of the transmission tree algorithm, this shows that when the number of distribution less than 500, the probability of relatively large number of cluster members is less than 3 , so the average location error is larger, with the increase of the nodes in the number of distribution , the tracking error will stabilize. The simulation results show that the proposed algorithm compared with the transmission tree algorithm has certain advantages in the tracking error.

C. The comparison of network energy consumption

On the basis of the same node energy consumption, detection radius of the classification algorithm of the network node average energy consumption comparison chart. As it shown in Figure 9, red line based algorithm the network lifetime curve, the green line tracking algorithm for network life curve for the tree structure of the traditional transmission. It can be seen that when the number of distribution is small, due to appear to track the target is lost, the use of high-power detection radius, energy consumption is relatively larger, but this can guarantee the target accurately track. When the distribution number is greater than 500, the algorithm average energy consumption of sensor nodes will stabilize. It can be seen that the average life span of the network in this algorithm is longer than the traditional algorithm, there is a great advantage in network energy consumption and target accurately track. Through the above simulation it can be seen that either 
uniform or variable motion, the algorithm can effectively track the target. Using the algorithm of dynamic cluster mechanism, the nodes in every moment to participate in the tracking is optimized between residual energy and location. When a smaller number of network is distributed, the nodes use a high-power detection radius, and it can efficiently ensure the accurate tracking, When the number of distribution reach a certain threshold and then the node use the low-power detection radius, and most of the nodes are in a dormant state, it can either ensure the accuracy of target tracking, and also significantly reduce energy consumption. Compared with the traditional tracking algorithm, the network lifetime can be significantly improved.

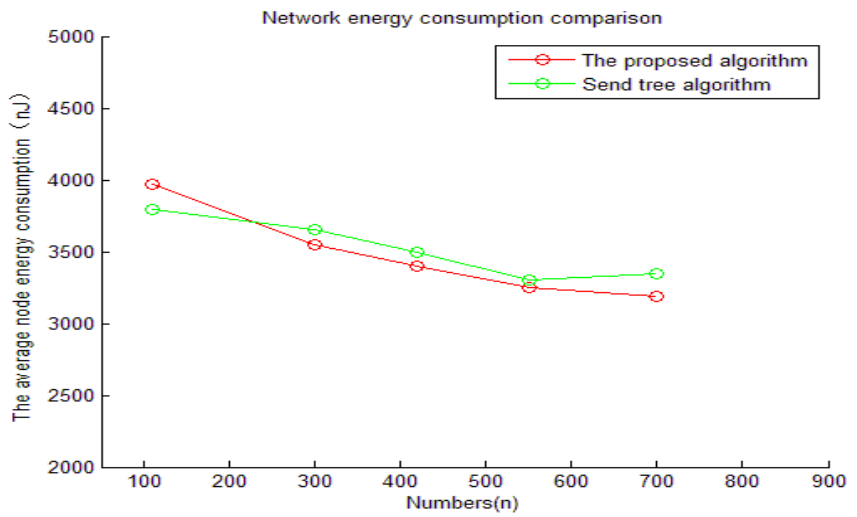

Figure 9. Network node average energy consumption comparison

\section{Conclusions}

In this paper, with the depth analysis of the characteristics of wireless sensor networks, target tracking technology, as well as several classical target tracking algorithm, we give a target tracking algorithms based on dynamic cluster mechanism and energy optimization. The algorithm considers both the node residual energy and the target RSSI signal strength factor in the clustering, each sensor node has two detection $\operatorname{radius}(\mathrm{r}<\mathrm{R})$. When the initial cluster created, relevant nodes in accordance with the node residual energy and the target signal strength choose whether or not to join the initial cluster, cluster structure will be dynamically adjusted as the target moves, and real-time to wake up to the corresponding node to keep track of the target.

When the tracking target is lost, it is time to start high-power detection radius of the sensor nodes to continue tracking the target, so we can reduce energy consumption at the same time, as far as possible to achieve the target tracking. Matlab simulation results show that the algorithm can guarantee the tracking accuracy, reduce energy consumption and prolong the network lifetime.

\section{References}

[1] I. Akyildiz, W. Su, Y. Sanakarasubramaniam, "Wireless sensor networks, A survey," Computer Networks, 2002, 38(4). pp. 393-422

[2] Frey H, Gorqen D. Geographical cluster-based routing in sensing-covered networks[J]. IEEE Trans on Parallel and Distributed Systems, 2006, 17(9): 899-911

[3] Estrin D, Girod L, Pottie G, Srivastava M, "Instrumenting the World with Wireless Sensor Networks" Proceedings of the international Conference on Acoustics, Speech and Signal Processing, pp. 233-239

[4] Zibo He, Research on Target Tracking and Energy Consumption Optimization Based on Dynamic Clusters in WSN, Shandong University 2013

[5] Qiang Ma, Guoqiang Yan, Energy Consumption Improvement of S-MAC Protocol in Wireless Sensor Networks [J]. Modern Electronics Technique.2011 ( 07 ) 